\title{
A juxtaposition of safety outcomes between various doses of sodium- glucose co-transporter inhibitors, in insulin-treated type-1 diabetes mellitus patients: a protocol for systematic review and meta-analysis of randomized controlled trials
}

\author{
Sumanta Saha ${ }^{1^{*}}$
}

\begin{abstract}
:
Background: Several clinical trials have tested the safety profile of sodium-glucose co-transport inhibitors' (SGLTis) in adult type 1 diabetes mellitus (T1DM) patients. However, no systematic review has yet compared its variation between large and low dose SGLTis. Henceforth, a review protocol is proposed here to review it.

Methods: Different electronic databases will be searched for randomized-controlled trials (published in the English language) studying the above objective, irrespective of their publication date. After selecting the eligible trials, their data on the study design, population characteristics, compared interventions, and outcomes of interest will be extracted. Then, utilizing the Cochrane tool, each trial's risk of selection bias, detection bias, performance bias, attrition bias, reporting bias, and other bias will be judged. Next, depending on clinical heterogeneity among the trials, a random-effect or fixed-effect model meta-analysis will be used to compare the respective outcomes. Via the Chi2 and 12 statistics, the statistical inconsistency among the trials will be estimated. When this is substantial, subgroup analysis will follow. Publication bias will be evaluated by funnel plots and Egger's test. A sensitivity analysis will be done to check different assumptions. If a quantitative juxtaposition is not possible, a narrative reporting will ensue.
\end{abstract}

Conclusion: The proposed study will perform a dose-wise juxtaposition of the safety profile of SGLTis in insulintreated T1DM patients.

Registration: Prospective Register of Systematic Reviews (PROSPERO) (Registration no. CRD42019146578)

Keywords: Type 1 Diabetes, Sodium-Glucose Transporter 1, Sodium-Glucose Transporter 2, Drug-Related Side

Effects and Adverse Reactions, Randomized Controlled Trial

\section{Background}

The autoimmune destruction of insulin (an anabolic hormone) producing pancreatic beta-cells leads to type 1 diabetes mellitus (T1DM), a disease characterized by hyperglycemia [1,2]. Although the exact etiology of the disease is unknown, the genetic predisposition may have some role. Individuals with certain human leukocyte antigen alleles (DR and DQ) are at greater risk of developing T1DM upon exposure to various trigger agents like viruses, environmental toxins, and dietary factors [1]. T1DM frequently begins between 4-6 and 10-14year-olds, although it can start at any age [1]. About 5-10\% of

*Correspondence: sumanta.saha@uq.net.au

${ }^{1}$ Independent researcher

Full list of author information is available at the end of the article diabetes patients are T1DM patients [1]. Complications of T1DM often include neuropathy, nephropathy, retinopathy, hypoglycemia, diabetic ketoacidosis, cardiomyopathy, and diabetic foot disease [1]. To prevent these hyperglycemic complications in T1DM patients, insulin, the mainstay of treatment, is required lifelong [1-3].

However, this entire insulin dependence doesn't suit every T1DM patient, because of the inconveniences of insulin therapy like multiple daily insulin injections and daily finger pricks to control and self-monitor the blood glucose levels, respectively.[4,5] Besides, there are adverse consequences of intensive insulin therapy on the health like lipohypertrophy or atrophy (when insulin is repeatedly injected at the same site), unwanted weight gain, raised risk of hypoglycemic episodes, and glycemic variations [6]. Some of these complications of 
absolute insulin therapy might affect the health and treatment of T1DM patients further. For instance, the subsequent injections in insulin-induced lipohypertrophied areas can slow down insulin absorption [6]. Likewise, the insulin-induced weight gain might raise the risk of cardiometabolic complications by decreasing compliance with the insulin regimen [6]. Therefore, to reduce the discomforts and complications of intensive insulin therapy, the role of insulin-independent adjunct therapeutics is vital in the treatment of T1DM patients. In this regard, sodiumglucose co-transport inhibitors (SGLTis) have drawn significant attention of the medical community.

SGLTis are phlorizin compounds [2,4]. By inhibiting sodium-glucose transporter 1 (SGLT1) and sodium-glucose transporter 2 (SGLT2) receptors, SGLTis cause glycosuria and decreased intestinal absorption of glucose [4]. SGLT2 receptors in the proximal convoluted tubule reabsorb almost $90 \%$ of the filtered glucose [6-8]. Examples of SGLT2 inhibitors include dapagliflozin, empagliflozin, and canagliflozin [9]. SGLT1 is predominantly found in the intestine, and its inhibition regulates the blood glucose level by increasing the release of gastrointestinal hormones through increased glucose delivery to the distal small intestine [6]. Sotagliflozin is a dual SGLT2/1 blocker; its capacity to inhibit SGLT1 receptors provides an additional benefit in decreasing the glucose levels postprandially [2].

Existing early stage trials suggested several efficacies of SGLTis in T1DM patients. Such trials comparing SGLT2 inhibitors (dapagliflozin: $5 \mathrm{mg}$ and $10 \mathrm{mg}$, empagliflozin: 25 $\mathrm{mg}, 10 \mathrm{mg}$, and $2.5 \mathrm{mg}$, and canagliflozin: $100 \mathrm{mg}$ and $300 \mathrm{mg}$ ) to placebo in insulin-treated inadequately controlled T1DM patients, demonstrated an improvement in glycemic control, body weight, and insulin requirement [10-12]. Likewise, trials that investigated sotagliflozin in T1DM patients and type 2 diabetes mellitus (T2DM) patients, observed improvement in glycemic control (by limiting the post-meal glycemic excursions), weight control, and daily insulin requirements [13]. A recent meta-analysis suggested that mega-dose empagliflozin treatment in insulin-treated T1DM patients with optimum renal functioning aids in achieving better glycemic control, compared to the placebo [14].

However, despite these benefits, due to safety concerns, both sotagliflozin [15] and SGLT2 inhibitors are currently not approved by the U.S. Food and Drug Administration [16]. Therefore, it is crucial to research the safety of SGLTis extensively. Contemporarily, several randomized controlled clinical trials have reported the safety profile of SGLTis in T1DM patients [12,17-19]. Although a statistical comparison of these side effects between the treatment arms was not available, based on its frequency, some idea about the safety profile of SGLTis can be made. The trials $[12,17,18]$ on T1DM patients that compared the safety profile between large and low dose empagliflozin, canagliflozin, dapagliflozin observed that the overall percentage of side-effects due to any cause were higher in the recipients of the former. In these large dose recipients this was $100 \%, 68 \%$, and $67 \%$ in empagliflozin $(25 \mathrm{mg})$, canagliflozin (300 mg), and dapagliflozin $(10 \mathrm{mg})$, respectively $[12,17,18]$. While the serious side effects did not happen with any dosages of empagliflozin in a trial [17], in dapagliflozin recipients, it was more frequent with the low dose $(5 \mathrm{mg}, 7 \%)$ than the large dose (10mg, 2.6\%) [18]. The serious side effects were found in $12.5 \%$ of T1DM patients using $400 \mathrm{mg}$ sotagliflozin in a trial [19]. In T1DM patients, the proportion of hypoglycemic side effects was dose irrespectively high in all canagliflozin users (98-99\%) [12], but in empagliflozin users, it varied dose-wise (2.5mg: 84\%, 10mg: 68\%, 25mg: 94\%) [17].

Given the safety concerns leading to non-approval of these drugs' use in T1DM patients by the US FDA and agglomeration of available clinical trials that tested their safety profile, it is imperative to synthesize novel evidence in this regard. Interestingly, the existing review articles have primarily compared the safety profile of SGLTis with the placebo [2023]. Although review attempts have been made to study the safety profile between large and low doses of specific SGLT2 inhibitors like empagliflozin and dapagliflozin [24,25], none have contrasted it across multiple SGLTis to synthesize summative evidence in this context. Therefore, to explore this under-reviewed area, we propose this systematic review and meta-analysis protocol.

\section{Methods}

The proposed review's inclusion criteria will be the following: a. Study design: Parallel-arm (any number of arms) randomized controlled trials. b. Participants: Adult (18 years or older) insulin-treated T1DM patients irrespective of their gender. The diagnosis of T1DM will be accepted as per the trialists. c. Intervention arm: The intervention groups should receive a mega-dose of dapagliflozin (10 mg), empagliflozin $(25 \mathrm{mg})$, canagliflozin $(300 \mathrm{mg})$, or sotagliflozin $(400 \mathrm{mg})$ every day. The determination of these mega doses was based on the maximum dose in which they were tested in T1DM patients in different clinical trials [12,17-19]. When the proposed review commences, if trials on any other SGLTis are found (besides those mentioned-above like ertugliflozin) matching the inclusion criteria, we will include those too in the same manner. d. Comparator arm: The comparator group/s should receive the same drugs as the intervention arm, however, at a lower dose. e. Outcome: Trials reporting side effects of SGLTis will be included. The definitions of these outcomes will be accepted as per the trialists.

The exclusion criteria will incorporate the following: a. Trial population diagnosed with type- 2 diabetes, gestational diabetes, or maturity-onset diabetes of young. b. Studies of other designs like crossover trials or quasi-experimental studies.

The proposed review protocol is registered in Prospective Register of Systematic Reviews (PROSPERO) (registration no. CRD42019146578) [26]. This protocol follows the PRISMA-P checklist [27].

\section{Search for eligible trials}

Next, the eligible trials' titles and abstracts will be searched in various electronic databases (PubMed, Embase, and Scopus). The search will not be limited to any date, but it will be restricted to the English language only.

The search strategy to be used in the PubMed database is described here. Following search terms will be used: 'safety' OR 'tolerance' OR 'adverse event' OR 'side effect' OR 'sideeffect' and 'canagliflozin' OR 'dapagliflozin' OR 'empagliflozin' OR 'sotagliflozin' OR 'sodium-glucose cotransporter' OR cotransporter* OR sglt* and 'type-1' OR 'type 1' and 'diabetes.' The search will be narrowed down to 
clinical trials by using the filter 'Randomized Controlled Trial' and 'Clinical Trial.' For other databases without such filters, instead, the following search terms will be used - 'trial' OR 'randomized' OR 'randomized' OR 'controlled.' Additionally, eligible trials will also be searched in the references of papers included in the proposed review.

\section{Selection of eligible trials}

The database search results will be uploaded to the Rayyan [28] systematic reviews software. Then, after excluding the duplicates, the titles and abstracts of papers will be read to find trials matching the above-mentioned eligibility criteria. A paper will be read in entirety if it seems to be eligible for inclusion in this review or when a decision of inclusion or exclusion cannot be made by reading the excerpts alone. The list of publications excluded after reading full-text will be retained with their reasons for elimination. The entire study selection process will adhere to the Prisma 2009 flow diagram [29].

When multiple trials source data from the same study population, one that counted the overall side effects (cause irrespective) based on the maximum number of side effects will be reviewed. If these latter are identical between trials, one with the longest follow-up time for adverse effects will be included in the review.

\section{Data extraction}

From the recruited trials, information about its details, participant characteristics, interventions compared, and outcomes of interest will be extracted. In trial details, its registration number, randomization method, blinding, duration, number of intervention arms, site (single centered or multicentric), participant consent, ethical clearance, country (where conducted), and funding information will be collected. The following participant characteristics will be gathered diagnosis, their number in each intervention arm, mean age, the average duration for which they have been diagnosed with T1DM, and their number for whom the outcome data of interest is not available. Concerning intervention information, the dosage and treatment regimen of each of the intervention arm will be collected. Finally, for the outcomes of interest, the number of participants experiencing it after taking at least one dose of the tested intervention will be extracted from each of the intervention groups.

\section{Risk of bias assessment}

For each trial, using the Cochrane tool, the risk of selection bias, performance bias, detection bias, attrition bias, reporting bias, and miscellaneous bias will be evaluated and categorized as low risk, high risk, or unclear (if it does not meet the low or high-risk categorization) [30].

The selection bias will be assessed by the random sequence generation method used to allocate interventions to the participants and the means used to conceal this allocation from the participants [30]. The performance bias will be based on the adequate blinding of study participants and personnel [30]. Depending on the blinding mechanism of the outcome assessors, the detection bias will be judged [30]. Based on the reasons and balance in missing outcome data between treatment arms, attrition bias will be evaluated [30,31]. By comparing the variation in a trial's reported findings from its protocol or prestated plans, the reporting bias will be assessed [30].

Author's roles

The review authors will independently do the literature search, data abstraction, and risk of bias assessment, and resolve conflict in opinions by discourse. If such disagreements sustain, a third-party opinion will be sought.

Meta-analysis

For binomial outcomes, risk ratios will be estimated. Whereas, for continuous outcomes, meta-analytic standardized or weighted mean difference estimation will use the endpoint means and its standard deviations (SD). If this endpoint SD for an outcome is unavailable in a trial, it will be substituted by the SD of the baseline mean of that outcome, and the meta-analysis will be performed using the mean changes and its SD changes from the baseline (using correlation coefficient 0.5). In trials with more than one intervention arms testing the low dosages, the respective outcome data will be combined across these treatment arms. The summary estimate from meta-analysis will be analyzed using either a random-effect model (Der Simonian and Laird method) or a fixed-effect model (inverse variance method). This model choice will depend on the clinical heterogeneity of the trials like trial settings, study design, participant characteristics, etc. and not the pre-determined statistical inconsistency. If the reviewed trials are clinically heterogeneous, a random-effect model will be used or viceversa.

A trial will be excluded from the meta-analysis if the outcome does not happen in both of the contrasting treatment arms. However, for dichotomous outcomes, when it happens in either of these arms, 0.5 will be added to each cell of the $2 \times 2$ table, and the trial will be incorporated in the meta-analysis.

For summary estimates of meta-analysis, a statistical significance will be determined at a $\mathrm{P}<0.05$ (and 95\% confidence interval). The trials with a high risk of bias will not be included in the meta-analysis. Any outcome for which a quantitative juxtaposition is not possible, a narrative reporting will ensue.

\section{Heterogeneity and publication bias}

Using the $I^{2}$ and $C h i^{2}$ statistics, the statistical inconsistency among the trials will be determined. At the $I^{2}$ statistics values of $0-40 \%, 30-60 \%, 50-90 \%$, and $75-100 \%$, the heterogeneity will be categorized as less, moderate, substantial, and considerable, respectively [30]. At a $P<0.1$, the statistical significance of the $C h i^{2}$ statistics will be estimated [30].

If considerable statistical heterogeneity is detected in a metaanalysis of at least 10 trials, a subgroup analysis will be done. It will be done between the trials testing SGLT2 inhibitors and the dual SGLT2/1 inhibitor, between the trials in which the participants' estimated glomerular filtration rate (GFR) was less than $60 \mathrm{~mL} / \mathrm{min} / 1.73 \mathrm{~m}^{2}$ and more than $60 \mathrm{~mL} / \mathrm{min} / 1.73 \mathrm{~m}^{2}$, and based on missing outcome data. Publication bias will be assessed using funnel plots. Additionally, an Egger's test will be used when at least ten trials are available for meta-analysis. Additional analysis

The following types of sensitivity analysis will be done by repeating the meta-analysis- a. By using a different meta- 
analysis model (fixed effect or random effect) then that was used during the preliminary analysis. b. By dropping one trial every time. c. By eliminating trials shorter than two weeks duration. d. By excluding trials that included trial population with GFR less than $60 \mathrm{~mL} / \mathrm{min} / 1.73 \mathrm{~m}^{2}$. e. If the mean changes and their SDs are used for any meta-analysis, the analysis will be iterated using a different correlation coefficient to determine the SD change (e.g., 0.8).

In the subgroup and sensitivity analysis proposed above, the rationale for using the benchmark of an optimum GFR (60 $\mathrm{mL} / \mathrm{min} / 1.73 \mathrm{~m}^{2}$ ) is that SGLT2 inhibitors is not recommended in T2DM patients with very low GFR [2]. Therefore, the notion is to see if such GFR plays any role in T1DM patients too.

Finally, for the dichotomous outcomes with statistically significant meta-analytic results, imputation case analysis (ICA) will be done to test the robustness of the preliminary analysis [32]. Assuming the event's occurrence and non-occurrence in all of the missing participants, the ICA-1 and ICA-0 analyses will be conducted, respectively. Moreover, the best- and worst-case scenario will be assessed along with the Gamble-Hollis analysis [33]. Statistically significant outcomes' will be graded for the quality of evidence using the Grades of Recommendation, Assessment, Development, and Evaluation (GRADE) Working Group's GRADE approach [34]. All analyses will be performed in Stata statistical software version 16 (StataCorp, College Station, Texas, USA).

\section{Discussion}

The chief implication of the prospective systematic review is that it will be one of the preliminary attempts to synthesize evidence in this context. It will perhaps help physicians, pharma companies, and relevant health authorities to understand how the side effects of SGLTis vary between different doses. Additionally, the proposed study's findings will help in comparing the results with similar research conducted on T2DM patients.

Regarding the strengths, evidence generated from the proposed review is likely to be rigorous since it will be based on randomized controlled trials (the highest level of epidemiological evidence). Its comprehensiveness is likely to be ensured due to not limiting the database search to any date range. Furthermore, the range of proposed sensitivity and imputation analysis will provide an idea of the robustness of the evidence generated from the review.

Despite these strengths, the suggested review is likely to have few weaknesses. At the review level, the inclusion of randomized controlled trials only limits its scope of reviewing studies of other designs like crossover trials or quasiexperimental studies, or good quality observational studies. Besides, limiting the database search to the English language literature only narrows down the scope of reviewing trials published (if any) in other languages. Lastly, since the diagnosis of T1DM and outcome definitions will be accepted as per the trialists, if the trials are extremely heterogenous in this context, the synthesized evidence may be at risk of bias.

\section{Conclusion}

The proposed review will compare the safety profile between high and low doses of SGLTis in insulin-treated T1DM patients.
Abbreviation

GFR: Glomerular filtration rate; GRADE: Grades of Recommendation, Assessment, Development, and Evaluation; ICA: Imputation case analysis; PROSPERO: Prospective Register of Systematic Reviews; SGLTis: Sodium-glucose co-transport inhibitors; SGLT1: Sodiumglucose transporter 1; SGLT2: Sodium-glucose transporter 2; SD: Standard deviations ; T1DM: Type 1 diabetes mellitus ; T2DM: Type 2 diabetes mellitus

Declarations

Acknowledgment

None

Funding

The author received no financial support for the research, authorship, and/or publication of this article.

Availability of data and materials

Data will be available by emailing sumanta.saha@uq.net.au

Authors' contributions

Sumanta Saha (SS) is the principal investigator of this manuscript (Review). SS is the responsible author for the study concept, design, writing, reviewing, editing, and approving the manuscript in its final form. SS has read and approved the final manuscript.

Ethics approval and consent to participate

We conducted the research following the Declaration of Helsinki. However, Review Articles need no ethics committee approval.

Consent for publication

Not applicable

\section{Competing interest}

The author declares that he has no competing interests.

Open Access

This article is distributed under the terms of the Creative Commons $\begin{array}{llll}\text { Attribution } & 4.0 & \text { International }\end{array}$ (http://creativecommons.org/licenses/by/4.0/), which permits unrestricted use, distribution, and reproduction in any medium, provided you give appropriate credit to the original author(s) and the source, provide a link to the Creative Commons license, and indicate if changes were made. The Creative Commons Public Domain Dedication waiver (http://creativecommons.org/publicdomain/zero/1.0/) applies to the data made available in this article, unless otherwise stated.

Author details

${ }^{1}$ Independent Researcher, sumanta.saha@uq.net.au

Article Info

Received: 29 July 2020

Accepted: 16 August 2020

Published: 20 August 2020

\section{References}

1. Lucier J, Weinstock RS. Diabetes Mellitus Type 1. StatPearls Treasure Isl StatPearls Publ [Internet]. 2020. Available from: http://www.ncbi.nlm.nih.gov/pubmed/29939535 
2. Fattah H, Vallon V. The Potential Role of SGLT2 Inhibitors in the Treatment of Type 1 Diabetes Mellitus. Drugs. 2018;78(7):717726. doi:10.1007/s40265-018-0901-y

3. Cherney DZ, Perkins BA, Soleymanlou N, Har R, Fagan N, Johansen $\mathrm{O}$, et al. The effect of empagliflozin on arterial stiffness and heart rate variability in subjects with uncomplicated type 1 diabetes mellitus. Cardiovasc Diabetol 2014; 13:28. Available from: http://cardiab.biomedcentral.com/articles/10.1186/14752840-13-28

4. Dellepiane S, Ben Nasr M, Assi E, Usuelli V, Letizia T, D’Addio $\mathrm{F}$, et al. Sodium glucose cotransporters inhibitors in type 1 diabetes. Pharmacol Res. 2018; 133:1-8. Available from: http://www.ncbi.nlm.nih.gov/pubmed/29689314

5. Alvarado-Martel D, Velasco R, Sánchez-Hernández RM, Carrillo A, Nóvoa FJ, Wägner AM. Quality of life and type 1 diabetes: a study assessing patients' perceptions and self-management needs. Patient Prefer Adherence 2015; 9:1315-23. Available from: http://www.ncbi.nlm.nih.gov/pubmed/26396503

6. Subramanian S, Baidal D, Skyler JS, Hirsch IB. The Management of Type 1 Diabetes [Internet]. Endotext. 2000. Available from: http://www.ncbi.nlm.nih.gov/pubmed/25905338

7. Boeder S, Edelman S V. Sodium-glucose co-transporter inhibitors as adjunctive treatment to insulin in type 1 diabetes: A review of randomized controlled trials. Diabetes, Obes Metab. 2019; 21:62$77 . \quad$ Available from: https://onlinelibrary.wiley.com/doi/abs/10.1111/dom.13749

8. Davidson JA. SGLT2 inhibitors in patients with type 2 diabetes and renal disease: overview of current evidence. Postgrad Med. 2019; 131:251-60. Available from: https://www.tandfonline.com/doi/full/10.1080/00325481.2019.16 01404

9. Australian Prescriber. New drug: Ertugliflozin for type 2 diabetes. 2019;42:68 Available from: http://care.diabetesjournals.org/lookup/doi/10.2337/dc18-S006

10. Mathieu C, Dandona P, Phillip M, Oron T, Lind M, Hansen L, et al. Glucose Variables in Type 1 Diabetes Studies with Dapagliflozin: Pooled Analysis of Continuous Glucose Monitoring Data From DEPICT-1 and -2. Diabetes Care 2019; 42:1081-7. Available from: http://care.diabetesjournals.org/lookup/doi/10.2337/dc18-1983

11. Rosenstock J, Marquard J, Laffel LM, Neubacher D, Kaspers S, Cherney DZ, et al. Empagliflozin as Adjunctive to Insulin Therapy in Type 1 Diabetes: The EASE Trials. Diabetes Care 2018; 41:2560-9. Available from: http://care.diabetesjournals.org/lookup/doi/10.2337/dc18-1749

12. Henry RR, Thakkar P, Tong C, Polidori D, Alba M. Efficacy and Safety of Canagliflozin, a Sodium-Glucose Cotransporter 2 Inhibitor, as Add-on to Insulin in Patients with Type 1 Diabetes. Diabetes Care 2015; 38:2258-65. Available from: http://care.diabetesjournals.org/lookup/doi/10.2337/dc15-1730

13. Sims H, Smith KH, Bramlage P, Minguet J. Sotagliflozin: a dual sodium-glucose co-transporter-1 and -2 inhibitor for the management of Type 1 and Type 2 diabetes mellitus. Diabet Med. 2018; 35:1037-48. Available from: http://www.ncbi.nlm.nih.gov/pubmed/29637608

14. Saha S, Saha S. A systematic review and meta-analysis of randomized controlled trials, juxtaposing the control of glycemia and blood pressure between large dose empagliflozin and placebo among type 1 diabetes patients. Int J Health Sci (Qassim). 2020;
14:40-52. Available from: https://www.ijhs.org.sa/index.php/journal/article/view/4346

15. Reuters. FDA rejects Sanofi-Lexicon add-on pill for type 1 diabetes. Health (Irvine. Calif). [cited 2020 Apr 23]. Available from: https://in.reuters.com/article/us-sanofi-fr-fda/fda-rejectssanofi-lexicon-add-on-pill-for-type-1-diabetes-idINKCN1R32DM

16. U.S. Food and Drug Administration. Sodium-glucose Cotransporter-2 (SGLT2) Inhibitors [Internet]. 2018 [cited 2019 Dec 26]. Available from: https://www.fda.gov/drugs/postmarketdrug-safety-information-patients-and-providers/sodium-glucosecotransporter-2-sglt2-inhibitors

17. Pieber TR, Famulla S, Eilbracht J, Cescutti J, Soleymanlou N, Johansen OE, et al. Empagliflozin as adjunct to insulin in patients with type 1 diabetes: a 4-week, randomized, placebo-controlled trial (EASE-1). Diabetes, Obes Metab. 2015; 17:928-35. Available from: http://doi.wiley.com/10.1111/dom.12494

18. Mathieu C, Dandona P, Gillard P, Senior P, Hasslacher C, Araki E, et al. Efficacy and Safety of Dapagliflozin in Patients with Inadequately Controlled Type 1 Diabetes (the DEPICT-2 Study): 24-Week Results from a Randomized Controlled Trial. Diabetes Care. 2018; 41:1938-46. Available from: http://care.diabetesjournals.org/lookup/doi/10.2337/dc18-0623

19. Sands AT, Zambrowicz BP, Rosenstock J, Lapuerta P, Bode BW, Garg SK, et al. Sotagliflozin, a Dual SGLT1 and SGLT2 Inhibitor, as Adjunct Therapy to Insulin in Type 1 Diabetes. Diabetes Care. 2015; 38:1181-8. Available from: http://www.ncbi.nlm.nih.gov/pubmed/26049551

20. Saha S, Saha S. A Systematic Review and Meta-Analysis of Randomised Controlled Trials, Contrasting the Safety Profile between Sodium-Glucose Cotransporter-2 Inhibitors and Placebo in Type 1 Diabetes Mellitus Patients. Int J Diabetes Metab [Internet]. S. Karger AG; 2020;1-12. Available from: https://www.karger.com/Article/FullText/506366

21. Yamada T, Shojima N, Noma H, Yamauchi T, Kadowaki T. Sodium-glucose co-transporter-2 inhibitors as add-on therapy to insulin for type 1 diabetes mellitus: Systematic review and metaanalysis of randomized controlled trials. Diabetes, Obes Metab. 2018; 20:1755-61. Available from: https://onlinelibrary.wiley.com/doi/abs/10.1111/dom.13260

22. Saha S. An Appraisal of a Systematic Review and Meta-Analysis of Randomized Clinical Trials on the Efficacy and Safety of Sodium-Glucose Cotransporter-2 Inhibitors as an Adjunct to Insulin Therapy in Type 1 Diabetes Patients. Int J Diabetes Metab. S. Karger AG; 2019;1-1. Available from: https://www.karger.com/Article/FullText/502743

23. Lu J, Tang L, Meng H, Zhao J, Liang Y. Effects of sodiumglucose cotransporter (SGLT) inhibitors in addition to insulin therapy on glucose control and safety outcomes in adults with type 1 diabetes: A meta-analysis of randomized controlled trials. Diabetes Metab Res Rev. 2019; e3169. Available from: http://www.ncbi.nlm.nih.gov/pubmed/30974510

24. Saha S, Saha S. The comparison of efficacy and safety between different doses of empagliflozin in insulin-treated type 1 diabetes mellitus patients: a systematic review and meta-analysis protocol. J Diabetes Metab Disord. 2020; 19:545-50. Available from: http://link.springer.com/10.1007/s40200-020-00544-x

25. Saha S, Saha S. A Systematic Review and Meta-Analysis of Randomized Controlled Trials Comparing the Safety of 
Dapagliflozin in Type 1 Diabetes Patients. Iran J Heal Sci. 2020;8. Available from: http://jhs.mazums.ac.ir/article-1-708-en.html

26. Saha S, Saha S. Comparison between sodium-glucose cotransporter inhibitors of high dose and low dose, and with placebo in contemporary insulin-treated type-1 diabetes patients: a systematic review and meta-analysis of randomised controlled trials. [Internet]. PROSPERO 2019 CRD42019146578. [cited 2019 Dec 8]. Available from: https://www.crd.york.ac.uk/prospero/display_record.php?ID=CR D42019146578

27. Moher D, Shamseer L, Clarke M, Ghersi D, Liberati A, Petticrew $\mathrm{M}$, et al. Preferred reporting items for systematic review and metaanalysis protocols (PRISMA-P) 2015 statement. Syst Rev [Internet]. 2015; 4:1. Available from: https://systematicreviewsjournal.biomedcentral.com/articles/10.11 86/2046-4053-4-1

28. Ouzzani M, Hammady H, Fedorowicz Z, Elmagarmid A. Rayyan - a web and mobile app for systematic reviews. Syst Rev [Internet]. 2016; 5:210. Available from: http://systematicreviewsjournal.biomedcentral.com/articles/10.118 6/s13643-016-0384-4

29. Liberati A, Altman DG, Tetzlaff J, Mulrow C, Gøtzsche PC, Ioannidis JPA, et al. The PRISMA statement for reporting systematic reviews and meta-analyses of studies that evaluate health care interventions: explanation and elaboration. J Clin
Epidemiol [Internet]. 2009;62: e1-34. Available from: https://linkinghub.elsevier.com/retrieve/pii/S0895435609001802

30. Higgins JPT GS (editors). Cochrane Handbook for Systematic Reviews of Interventions Version 5.1.0 [updated March 2011]. [Internet]. Cochrane Collab. 2011. Available from: www.cochrane-handbook.org

31. Saha S. Impact of missingness on clinical trials on the effectiveness of antenatal vitamin D supplementation in gestational diabetes mellitus patients. J Ideas Heal [Internet]. 2020; 3:138-9. Available from: https://www.jidhealth.com/index.php/jidhealth/article/view/47

32. Higgins JP, White IR, Wood AM. Imputation methods for missing outcome data in meta-analysis of clinical trials. Clin Trials J Soc Clin Trials [Internet]. 2008; 5:225-39. Available from: http://journals.sagepub.com/doi/10.1177/1740774508091600

33. Gamble C, Hollis S. Uncertainty method improved on best-worst case analysis in a binary meta-analysis. J Clin Epidemiol [Internet]. 2005; 58:579-88. Available from: https://linkinghub.elsevier.com/retrieve/pii/S0895435605000417

34. 34. Atkins D, Best D, Briss PA, Eccles M, Falck-Ytter Y, Flottorp $\mathrm{S}$, et al. Grading quality of evidence and strength of recommendations. BMJ [Internet]. 2004; 328:1490. Available from:

http://www.bmj.com/lookup/doi/10.1136/bmj.328.7454.1490 